\title{
SILICON ACCELEROMETER WITH DIFFERENTIAL FREQUENCY MODULATION AND CONTINUOUS SELF-CALIBRATION
}

\author{
Alexander A. Trusov, Sergei A. Zotov, Brenton R. Simon, Andrei M. Shkel \\ MicroSystems Laboratory, University of California, Irvine, CA, USA
}

\begin{abstract}
We report a new silicon MEMS accelerometer based on differential Frequency Modulation (FM) with experimentally demonstrated self-calibration against dynamic temperature environment and $\mu \mathrm{g}$-level Allan deviation of bias. The sensor architecture is based on resonant frequency tracking in a vacuum packaged SOI tuning fork oscillator with a high Q-factor. The oscillator is instrumented with a DC voltage biased parallel plate capacitor, which couples the proof mass displacement to the effective stiffness by means of the negative electrostatic spring effect. External acceleration is detected as an FM signal. To address drift over temperature, the MEMS sensor die incorporates two identical tuning forks with opposing axes of sensitivity. Demodulation of the differential FM output from the two simultaneously operated oscillators eliminates common mode errors and provides a continuously self-calibrated FM output. An $\mathrm{x}$-axis SOI prototype with a tunable scale factor was built and characterized over dynamic temperature environment, experimentally demonstrating continuous self-calibration.
\end{abstract}

\section{INTRODUCTION}

While silicon MEMS accelerometers have proven themselves as commercially successful devices, significant challenges remain in bringing them to high performance, mission critical applications. Conventional micromachined pendulous accelerometers operate as analogue Amplitude Modulated (AM) systems, with an inherent gain-bandwidth tradeoff and dynamic range limited by the stability of capacitive pickoff electronics. These analogue devices typically show poor long term and environmental stability. Packaging requirements for the highly damped pendulous accelerometers contradict the vacuum sealing requirements of high performance MEMS gyroscopes, complicating single die integration.

Another inherent disadvantage of conventional MEMS sensors using amplitude modulated signals comes from the limited dynamic range, the ratio between the full scale linear range and the smallest detectable input stimulus change. In the best case scenario, AM capacitive readout with carefully selected low-noise electronic components can only achieve a dynamic range of $10^{\wedge} 6$, with a practical limit of $10^{\wedge} 5$. This means that achieving a better than $10^{\wedge} 6$ dynamic range and $1 \mathrm{ppm}$ stability (requirement of the navigation grade) is practically impossible with conventional MEMS sensors architectures. These fundamental limitations on the dynamic range and output stability prevent the use of MEMS gyroscopes and accelerometers in many important applications.

An alternative approach to resolving these limitations is using a frequency modulated accelerometer, where induced acceleration changes the resonant frequency of the device due to changes in the total effective stiffness $[1,2]$.

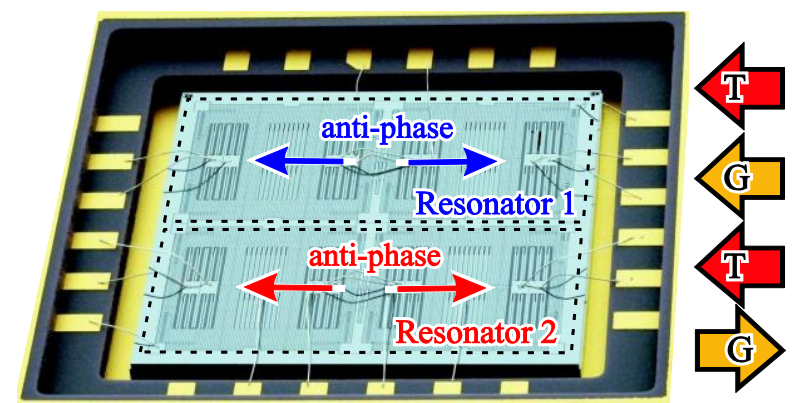

Figure 1: Photograph of a differential FM accelerometer fabricated using an in-house $100 \mu \mathrm{m}$ SOI process. Arrows show sensitivity to acceleration and temperature.

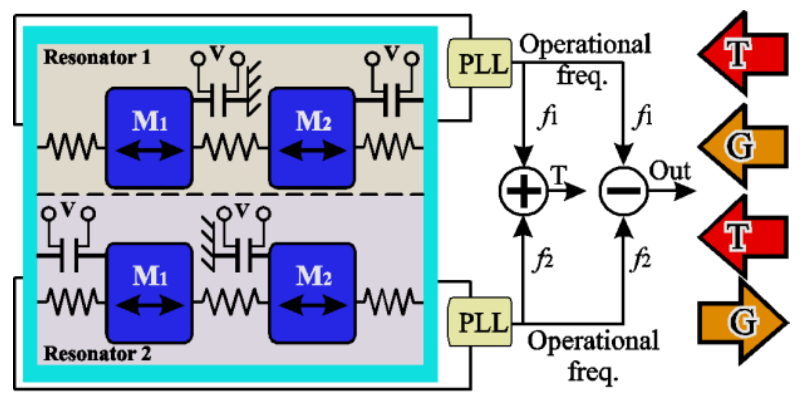

Figure 2: Concept of the differential FM accelerometer with temperature self-calibration. Arrows show axes of sensitivity to external acceleration and temperature.

Performance of previously reported FM accelerometers is limited by relatively low Q-factors and temperature dependency. The main challenge to overcome in silicon MEMS accelerometers with FM operation is temperature sensitivity of the resonant frequency, caused by the strong temperature dependency of the silicon's Young's modulus.

In this paper we propose a wide dynamic range, differential FM accelerometer architecture with tunable scale factor and inherent self-calibration against dynamic environment changes, Figure 1. The differential FM accelerometer approach relies on tracking of the resonant frequencies of two high-Q mechanical MEMS oscillators to produce quasi-digital and decoupled FM measurements of the input acceleration and temperature, Figure 2.

\section{SENSOR CONCEPT AND DESIGN \\ Principle of Operation}

The proposed differential FM accelerometer consists of two identical silicon MEMS tuning fork resonators. Each of the two resonators has two mechanical degrees of freedoms: in-phase and anti-phase motion of the coupled tines. The anti-phase mode of the resonator is dynamically balanced, eliminating dissipation of energy due to linear and angular vibrations of the substrate. Increase of the Q-factor up to the fundamental thermoelastic limit improves precision, stability, and phase noise for the anti-phase vibration. In contrast, the in-phase vibration has a low Q-factor, which is limited by the anchor loss [3]. 


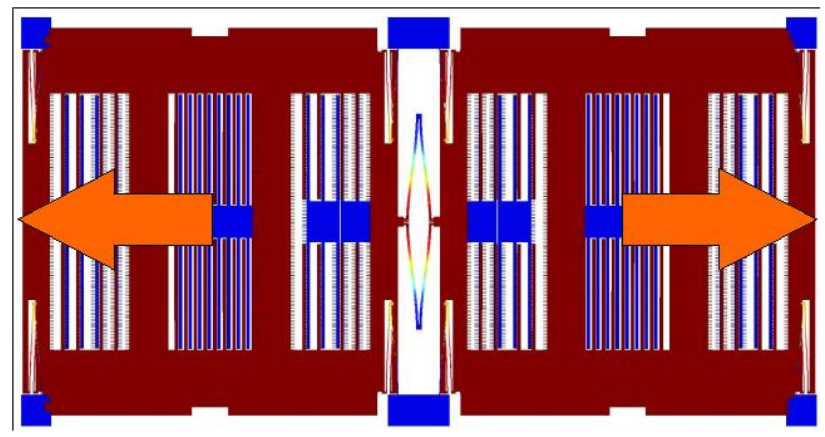

(a) Anti-phase mode shape at $2.6 \mathrm{kHz}, Q_{T E D}=0.3$ million.

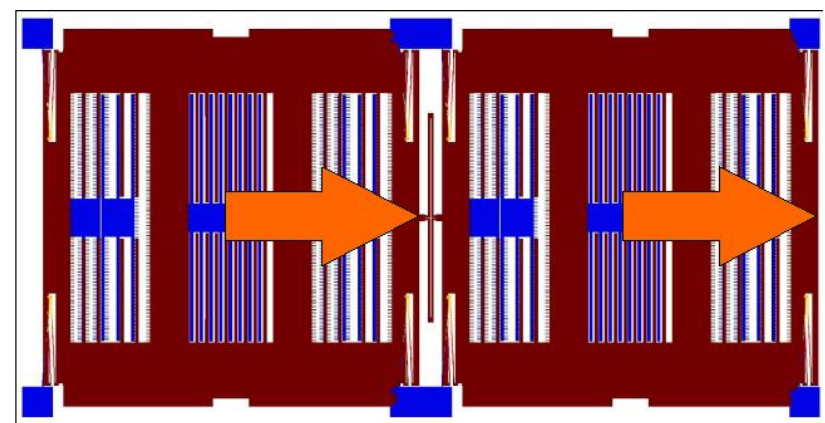

(b) In-phase mode shape at $0.9 \mathrm{kHz}$.

Figure 3: Finite Element Modeling (FEM) results illustrating the (a) anti-phase and (b) in-phase vibratory modes of the FM accelerometer.

Each tine includes differential lateral comb electrodes for electrostatic excitation of the anti-phase mode, differential lateral comb electrodes for capacitive detection, and non-differential parallel plate capacitors for modulation of stiffness by means of the negative electrostatic spring effect. By applying a DC voltage bias on the parallel plates, a negative electrostatic spring is created, the stiffness of which is proportional to the square of the bias voltage and inversely proportional to the cube of the capacitive gap. This makes the anti-phase natural frequency highly sensitive to the gap between the fixed and moving parallel plate electrodes. In other words, the in-phase displacement of the two tines modulates the resonant frequency of the anti-phase mode.

The applied inertial acceleration produces the in-phase shift of proof masses, Figures 1, 2. This shift is detected by tracking the resonant frequency of the high Q-factor anti-phase mode. The relatively low Q-factor of the in-phase mode provides the short step response, and wide bandwidth of the accelerometer. At the same time, the high Q-factor of the balanced anti-phase mode guarantees high frequency resolution and stability, improving the accelerometer performance. The combination of the high Q-factor of the anti-phase mode and the low Q-factor of the in-phase mode eliminates the noise versus bandwidth tradeoff of conventional accelerometers. In addition, FM sensor architectures are known to be robust against mechanical and electromagnetic interferences $[4,5]$.

\section{Finite Element Modeling}

Finite Element Modeling (FEM) was completed using Comsol Multiphysics to determine the vibratory modes of this device. The 2-D model of the device consists of

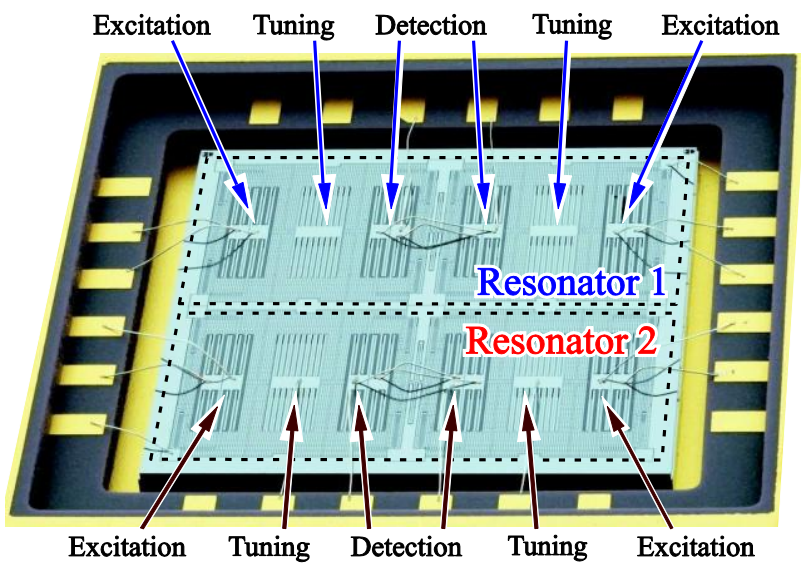

Figure 4: Photograph of a differential FM accelerometer fabricated using an in-house $100 \mu \mathrm{m}$ SOI process.

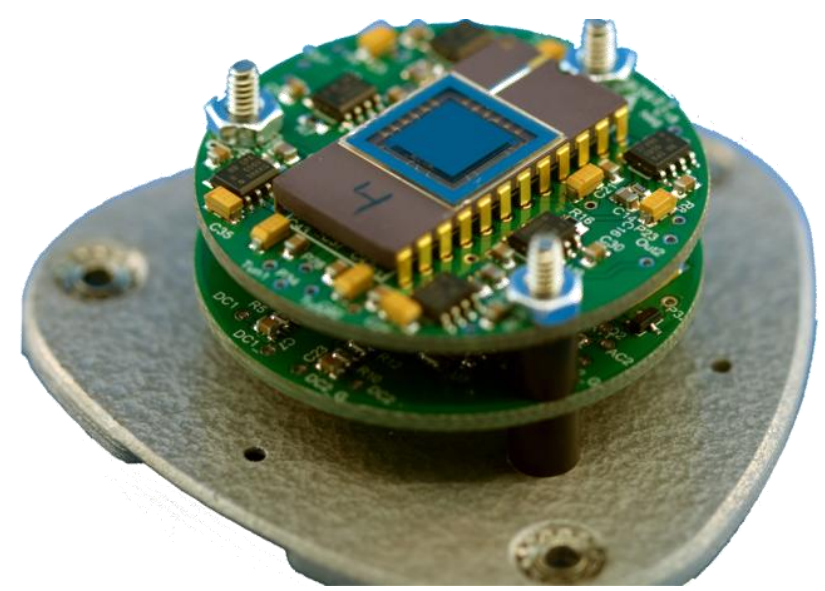

Figure 5: Photograph of a packaged differential FM accelerometer assembled with signal conditioning PCBs.

296,000 triangular mesh elements, the structure of which was imported from the lithography mask used to create the actual device, Figure 3. Because the device moves only along the $\mathrm{x}$-axis and is fabricated from single crystalline silicon, a uniform Young's Modulus was used with a value of $160 \mathrm{GPa}$. The in-phase and anti-phase resonance frequency were found to be $0.9 \mathrm{kHz}$ and $2.6 \mathrm{kHz}$, respectively. Through the suspension system design, the next mode of vibrations was pushed to $25 \mathrm{kHz}$ frequency to minimize cross axis sensitivity. A second FEM model was then executed to analyze the fundamental thermoelastic limit of the Q-factor. For the anti-phase mode of vibrations, a Q-factor of 0.3 million was predicted.

\section{Self-Calibration Through Differential FM}

The proposed temperature self-calibration approach takes advantage of the differential design, in which both oscillators have the same sensitivity to temperature but opposite sensitivity to external acceleration, Figures 1, 2. The dependency of frequency on temperature has a well known linear relationship for single crystalline silicon, enabling direct self-sensing of temperature. The differential FM signal processing tracks the frequency difference between the two resonant accelerometers, enabling drift free measurement of acceleration, Figure 2. In this approach, the FM accelerometer provides a quasi-digital measurement of the input acceleration as well 


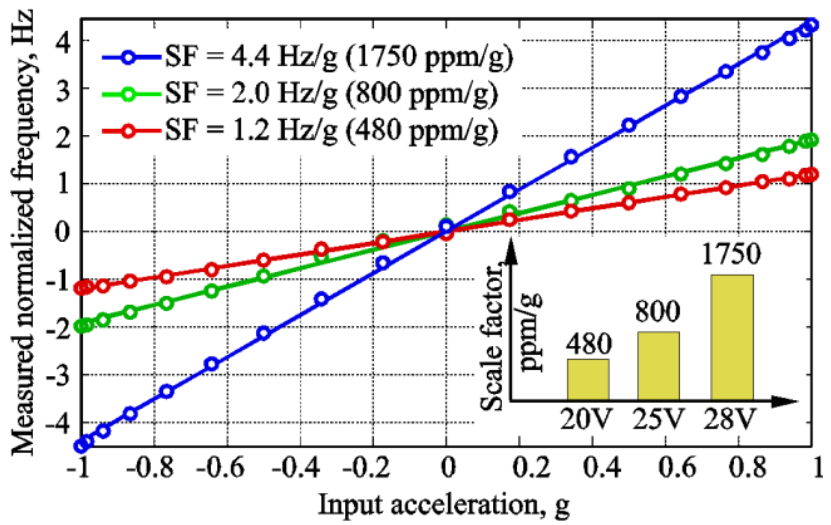

Figure 6: Measured input-output characteristic of FM accelerometer for different stiffness modulation DC voltages. Inset: scale factor vs. modulation DC voltage.

as direct measurement of the accelerometer temperature. The sensor becomes its own thermometer, eliminating thermal lags and hysteresis typical in compensation schemes using an external temperature sensor.

\section{CHARACTERIZATION RESULT Prototype Fabrication and Packaging}

The fabrication of prototype FM accelerometers was performed using an in-house, wafer-level, single mask process. Devices were fabricated using Silicon-on-Insulator (SOI) wafers with a $100 \mu \mathrm{m}$ single crystalline silicon device layer, a $5 \mu \mathrm{m}$ buried oxide layer, and a $500 \mu \mathrm{m}$ handle wafer, Figure 4. After wafer fabrication and dicing, sensors were attached to a ceramic DIP-24 package, wirebonded, and vacuum sealed in-house at $\sim 1$ Torr. In future fabrication runs, accelerometers will be vacuum sealed at 0.1 mTorr using getter to enable ultra-high Q-factor operation. For testing, the packaged sensors were assembled with signal conditioning electronics, Figure 5.

\section{Scale Factor Characterization}

A standard multi-point tumble test was carried out for a single tuning fork (non-differential) FM accelerometer using an Ideal Aerosmith 2102 Series Two-Axis Position and Rate Table System. The sensor was tested by measuring the change of the anti-phase resonant frequency as a function of inclination angle with $10^{\circ}$ increments. The resonance frequency of the accelerometer was recorded for each orientation within a range from $-\mathrm{g}$ to $\mathrm{g}$. This experiment was performed for three different tuning voltages $(28,25$ and $20 \mathrm{~V})$, revealing linear response to acceleration with tunable scale factors of 4.4, 2.0 and 1.2 $\mathrm{Hz} / \mathrm{g}$, respectively, Figure 6.

\section{Self-Calibration}

To evaluate the proposed self-calibration concept, a differential FM accelerometer with two tuning fork oscillators was paced into a TestEquity 107 temperature chamber. The temperature was set to $70{ }^{\circ} \mathrm{C}$ for the duration of 3 hours. The temperature control was then turned off and the output signals from both tuning forks were recorded, Figure 7. Each oscillator showed an identical $500 \mathrm{mg}$ drift over the temperature change. Differential FM

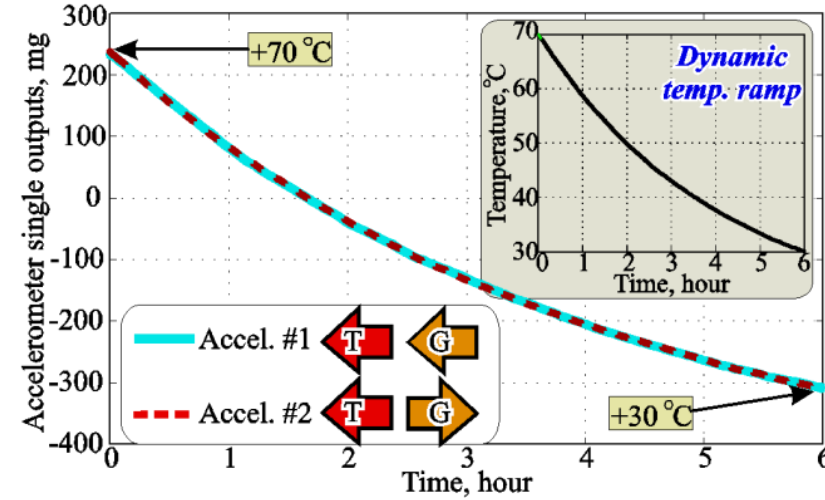

Figure 7: Measured output of two differential FM channels during dynamic temperature ramp. Bias drifts track each other, enabling self-calibration.

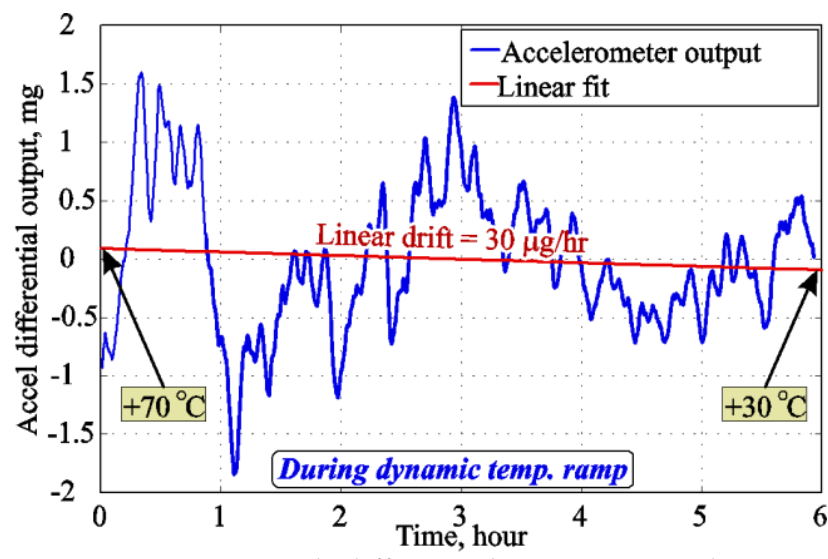

Figure 8: Measured differential FM output during a dynamic temperature ramp, showing self-calibration against temperature with a low drift rate of $30 \mu \mathrm{g} / \mathrm{hr}$.

demodulation provided automatic calibration against temperature by canceling common frequency drifts between the two sensors. As shown in Figure 8, the drift over temperature was reduced to approximately $1 \mathrm{mg}$, currently limited by the noise performance of oscillators sealed with 1 Torr pressure. Testing of differential FM accelerometers sealed with getter is expected to improve the bias several orders of magnitude.

Self-calibration by differential FM also applies to the scale factor. The anti-phase resonant frequencies of both tuning fork oscillators were characterized as functions of applied acceleration at two different temperatures of $30{ }^{\circ} \mathrm{C}$ and $75^{\circ} \mathrm{C}$, Figure 9 (a). The measured frequency split between the nominally equal modal frequencies was proportional to the input acceleration, Figure 9(b). Without any active temperature compensation, experimental characterization of the FM accelerometer at $30{ }^{\circ} \mathrm{C}$ and 75 ${ }^{\circ} \mathrm{C}$ revealed less than 0.5 percent response fluctuation (within the accuracy of the experimental setup) despite a 4 $\mathrm{Hz}$ drop of the nominal frequency, Figure 9(b).

\section{Noise Performance}

Allan deviation analysis of FM accelerometer in-run performance at constant temperature is shown in Figure 10. For a single tuning fork (non-differential) FM accelerometer, three regimes are identified: a $-1 / 2$ slope white noise of frequency for time constants of several 


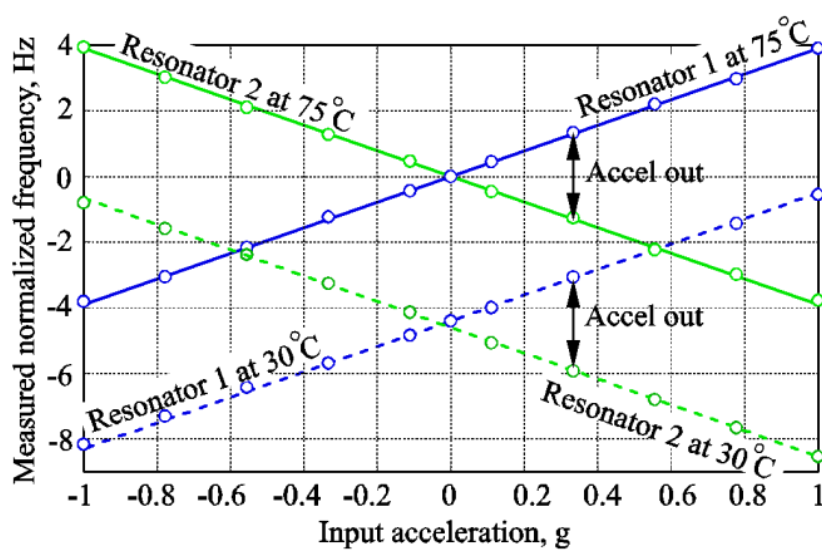

(a) Measured resonant frequencies $f_{1,2}$ as a function of the input acceleration for $30{ }^{\circ} \mathrm{C}$ and $75{ }^{\circ} \mathrm{C}$. Differential frequency split $f_{1}-f_{2}$ is invariant to temperature.

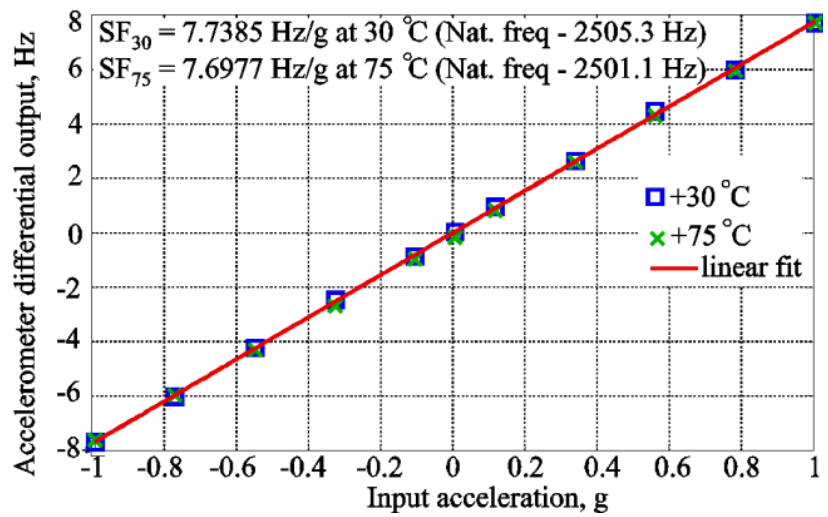

(b) Measured acceleration responses for $30^{\circ} \mathrm{C}$ and $75{ }^{\circ} \mathrm{C}$ using the differential frequency split.

Figure 9: Characterization of the differential FM accelerometer at $30{ }^{\circ} \mathrm{C}$ and $75{ }^{\circ} \mathrm{C}$, demonstrating self-calibration to temperature. There is less than $0.5 \%$ response fluctuation.

seconds, a zero slope flicker noise floor, and a +1 slope temperature ramp at time constants above 10 seconds. Differential FM demodulation using two tuning forks removes the +1 slope temperature ramp, revealing the bias instability of $6 \mu \mathrm{g}$ at $20 \mathrm{~s}$. In combination with the design linear range of $20 \mathrm{~g}$, the sensor demonstrates a wide dynamic range of $130 \mathrm{~dB}$ dynamic range.

\section{CONCLUSIONS}

A silicon MEMS accelerometer based on voltage controlled Frequency Modulation has been proposed and experimentally validated. The accelerometer employs a pair of two tuning fork oscillators with opposing axes of sensitivity to simultaneously measure the die temperature and external acceleration. Differential demodulation of the two FM outputs provides continuous self-calibration of the accelerometer against temperature change and other common mode effects. In contrast to conventional MEMS accelerometers, vacuum packaging is beneficial for the FM accelerometer, making it an attractive candidate for single die integration with high performance silicon MEMS Coriolis Vibratory gyroscopes (CVGs). Furthermore, single die integration of the FM accelerometer with the recently introduced FM gyroscope [6] is expected to pave

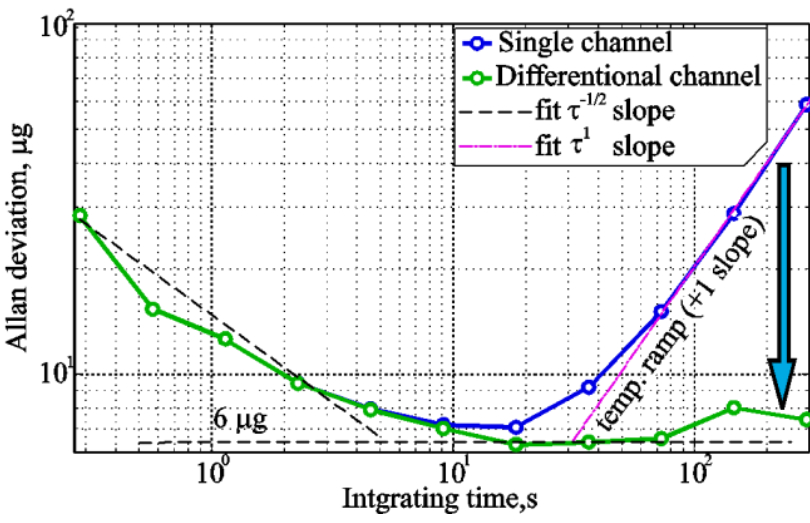

Figure 10: Measured Allan deviation for a vacuum sensor. Differential FM demodulation removes temperature ramp and achieves a $6 \mu \mathrm{g}$ bias at $20 \mathrm{sec}$.

the way for a high performance, wide dynamic range MEMS IMU with quasi-digital low power architecture and strong immunity against mechanical and electromagnetic interferences.

\section{ACKNOWLEDGEMENTS}

The work was supported by ONR and NSWCDD under grant N00014-11-1-0483 and DARPA and SPAWAR under contract N66001-12-C-4035 (Program Manager Dr. Tayo Akinwande). The accelerometers were designed, packaged, and experimentally characterized at the MicroSystems Laboratory, University of California, Irvine. MEMS fabrication was done at the UCI INRF and UCLA NRF.

\section{REFERENCES}

[1] R. Hopkins, et. al., "The silicon oscillating accelerometer: a high-performance MEMS accelerometer for precision navigation and strategic guidance applications," ION NTM 2005, 24-26 January 2005, San Diego, CA, pp. 970-979.

[2] S. Sung, J. G. Lee, T. Kang. "Development and test of MEMS accelerometer with self-sustained oscillation loop," Sensors and Actuators A 109, 2003, pp. 1-8.

[3] A.A. Trusov, A.R. Schofield, A.M. Shkel, "A substrate energy dissipation mechanism in in-phase and anti-phase micromachined $\mathrm{z}$-axis vibratory gyroscopes," JMM, vol. 18, pp. 095016(10), September 2008.

[4] A.A. Seshia, R.T. Howe, S. Montague, "An integrated microelectromechanical resonant output gyroscope," in: Proc. MEMS'02, 2002, pp. 722-726.

[5] C. Comi et al.,"A High Sensitivity Uniaxial resonant accelerometer," Proc. IEEE MEMS 2010, pp. 260-263.

[6] S.A. Zotov, A.A. Trusov, A.M. Shkel, "High-range angular rate sensor based on mechanical frequency modulation," IEEE/ASME JMEMS, vol. 21, no. 2, April 2012, pp. 398-405.

\section{CONTACT}

* A.A. Trusov, University of California, Irvine tel: +1-949-824-6314; alex.trusov@gmail.com. 